\title{
Acute Bilateral Renal Cortical Necrosis Complicating Pyomyoma
}

\author{
Katsutoshi Kuriyama ${ }^{1}$, Tetsuya Makiishi ${ }^{2}$, Sayako Maeda ${ }^{2}$, Takashi Konishi ${ }^{3}$ \\ and Kunihiko Hirose ${ }^{3}$
}

Key words: renal cortical necrosis, acute renal failure, acute tubular necrosis, pyomyoma

(Inter Med 49: 511-512, 2010)

(DOI: 10.2169/internalmedicine.49.3184)

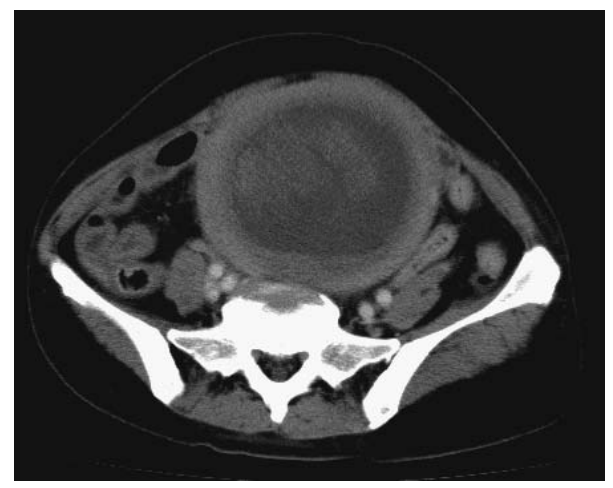

Picture 1A. A contrast-enhanced computed tomography (CT) scan of the abdomen showed a large cystic mass with ring enhancement originated from uterus.

A 51-year-old woman was admitted to our hospital complaining of worsening fever, chills, and low back pain over the previous 6 hours. Vital signs showed temperature of 38.3 ${ }^{\circ} \mathrm{C}$, pulse rate of 120 beats per minute, blood pressure of $140 / 72 \mathrm{mmHg}$, and respiratory rate of 24 per minute. Physical examination revealed a large mass occupying the middle and lower portions of the abdomen, which had been diagnosed as uterine myoma one month before. Blood tests on admission revealed that her white cell count was $15,900 / \mu \mathrm{L}$ with left shift, C-reactive protein was $13.1 \mathrm{mg} / \mathrm{dL}$, and serum creatinine level was $0.71 \mathrm{mg} / \mathrm{dL}$. The patient became anuric on the day of admission.

A contrast-enhanced computed tomography (CT) scan of the abdomen was performed to investigate a focus of infection. The CT scan showed a large cystic mass with ring enhancement originating from the uterus, suggesting pyomyoma, a fatal complication of uterine myoma (Picture 1A).

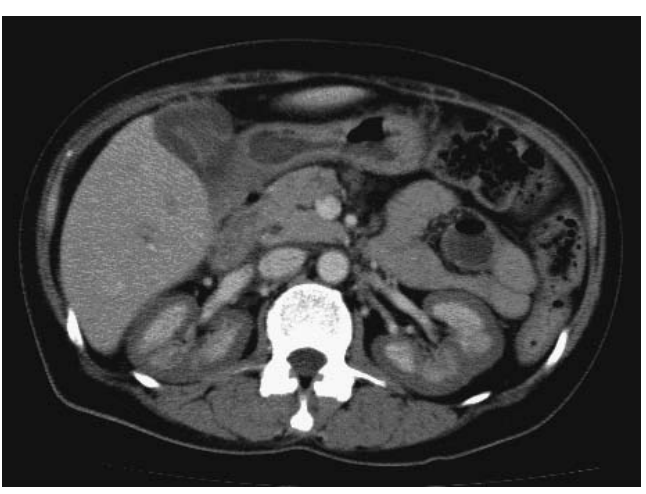

Picture 1B. The CT scan showed lack of bilateral renal cortical enhancement sparing thin rim of subcapusular cortex, juxtamedullary and medullar areas, characteristic findings of renal cortical necrosis.

The CT scan also showed diffuse and bilateral cortical hypodense areas surrounded by capsular enhancement in both kidneys, diagnostic finding of bilateral renal cortical necrosis (Picture 1B).

Total hysterectomy was performed on the 3rd day of admission. Gram stain of the abscess contents revealed numerous white blood cells and gram-negative rods, but culture of the abscess contents showed no growth, probably due to prior use of antibiotics. General improvement was noted after surgery. Her daily urine output has increased up to 500 to $750 \mathrm{ml}$, but she remained dialysis-dependent.

Bilateral acute renal cortical necrosis is a rare but severe form of acute tubular necrosis characterized by necrosis of the renal cortex but sparing of the medulla. It accounts for only $2-4 \%$ cases of acute renal failure in western countries $(1,2)$. It is usually associated with obstetric complication, but other disorders, such as poisoning, acute pancreati-

${ }^{1}$ Department of Gastroenterology, Otsu Red Cross Hospital, Otsu, ${ }^{2}$ Department of Cardiology, Nephrology Division, Otsu Red Cross Hospital, Otsu and ${ }^{3}$ Department of Cardiology, Otsu Red Cross Hospital, Otsu

Received for publication November 24, 2009; Accepted for publication November 25, 2009

Correspondence to Dr. Tetsuya Makiishi, makiishi@otsu.jrc.or.jp 
tis, or sepsis can also cause it (2). Massive bilateral renal cortical necrosis typically occurs in the setting of hypovolemia and endotoxic shock. Dialysis is required in most survivors (2).

The diagnosis is best made by CT scan, especially during the initial phase of the disease. The characteristic finding is the lack of renal cortical enhancement sparing thin rim of the subcapusular cortex, juxtamedullary and medullar areas (3).

\section{References}

1. Chugh KS, Jha V, Sakhuja V, Joshi K. Acute renal cortical necrosis: a study of 113 patients. Ren Fail 16: 37-47, 1994.

2. Prakash J, Vohra R, Wani IA, et al. Decreasing incidence of renal cortical necrosis in patients with acute renal failure in developing countries: a single-centre experience of 22 years from Eastern India. Nephrol Dial Transplant 22: 1213-1217, 2007.
3. Suzer O, Shirkhoda A, Jafri SZ, Madrazo BL, Biz KG, Mastromatteo JF. CT features of renal infarction. Eur J Radiol 44: 59-64, 2002.

(C) 2010 The Japanese Society of Internal Medicine http://www.naika.or.jp/imindex.html 